\title{
Determination of Clozapine by Air Assisted Dispersive Liquid- Liquid Microextraction Based on Solidification of Organic Droplet Followed by HPLC in Human Serum
}

\author{
Amir Hosein Mohammad Shafiee a, Mohammad Reza Mohammad Shafiee ${ }^{b, *}$ \\ a The Village High School, 13051 Whittington Drive, Houston, Texas, United States, P.O. Box: 77077 \\ ${ }^{b}$ Faculty of Sciences, Najafabad Branch, Islamic Azad University, Najafabad, Esfahan, Iran, P.O. Box: 517
}

\section{ARTICLE INFO}

Received: 24 May 2019

Revised: 14 July 2019

Accepted: 19 August 2019

Available online: 19 August 2019

\section{K E Y W O R D S}

\section{Clozapine}

Air assisted dispersive liquid-liquid

Microextraction

Solidification of organic droplet

Serum analysis

\begin{abstract}
A B S T R AC T
Due to the challenges of maintaining serum levels in a safe therapeutic range (1$3 \mu \mathrm{M})$, Clozapine is one of the most promising medications for managing schizophrenia. Recently the barrier to the broader use of clozapine has been recognized to be the measurement of clozapine levels, which is however challenging due to the complexity of serum samples. The determination of clozapine is mainly conducted through a novel, simple, rapid and sensitive air assisted dispersive liquid-liquid microextraction based on solidification of organic droplet method that has been developed and validated by highperformance liquid chromatography with UV detection in human serum. In this method, due to the usage of an organic solvent with a low density and appropriate melting point, no microsyringe or fiber is required to support the organic microdrop. Furthermore, the extractant droplet can be collected easily by solidifying it at low temperature. 1-Undecanol was selected as extraction. The optimization of Parameters that influence extraction efficiency, i.e., volumes of extracting solvent, $\mathrm{pH}$, and salt effect, were performed using a factor by factor approach. Enrichment factor for clozapine under optimal conditions was 128. The limit of detection was $1.34 \mathrm{ng} / \mathrm{mL}$ in serum sample. The proposed method was successfully applied to the determination of clozapine in serum samples of a person under clozapine therapy.
\end{abstract}

\section{G R A P H I C A L ABS T RACT}

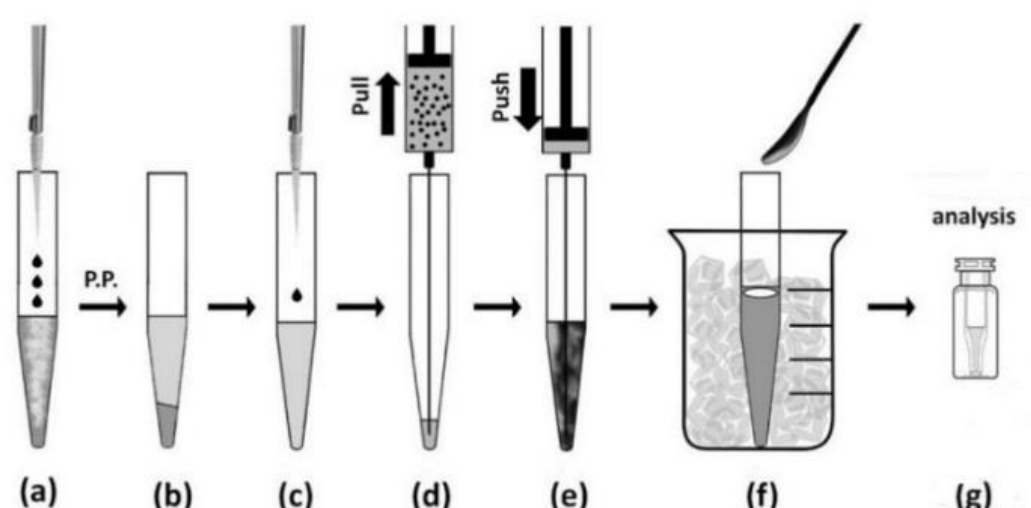

* Corresponding author's E-mail address: Shafiee.chem@gmail.com 


\section{Introduction}

The first "atypical antipsychotic" introduced in therapy was Clozapine (Clozaril or Leponex, 8-chloro-11-(4'-methyl)piperazine-5-H-dibenzo [b, e] [1,4]-diazepine, Figure 1). It has been used for the treatment of acute schizophrenia and manic psychosis. Clozapine (CLO) is an effective agent especially efficacious in patients suffering from treatment-resistant schizophrenia [1,2]. It is still the only antipsychotic agent with a significant reduction in all-cause mortality and suicidality $[3,4]$. compared to other antipsychotic therapies, Patients with schizophrenia on CLO are significantly more likely to function independently and maintain employment [5]. It is a serotonergic (5-HT2) antagonist and a dopaminergic (D2) antagonist, with affinity also toward histamine, adrenergic and cholinergic receptors [6]. Regarding classical neuroleptics, Clozapine has some advantages; it causes a lower rate of extrapyramidal side effects [7] and hyper prolactinemia [8], however it can cause severe agranulocytosis [9]. Cardiotoxicity, in the form of myocarditis and associated dilated cardiomyopathy, fatal agranulocytosis and toxic megacolon are significant adverse effects related to clozapine and can affect the utility of clozapine in the treatment of resistant schizophrenia, as these conditions may contribute to significant morbidity and mortality.

Therapeutic drug monitoring is a useful instrument for the clinical management of patients receiving a pharmacotherapy, particularly in psychiatry. For an optimal supervision of patient drug regimen, therapeutic drug monitoring antidepressants is necessary to avoid medical complications, intoxication, non-responsiveness or noncompliance. The general techniques for analyzing psychotropic drugs in different biological fluids are based on a combination of efficient separation method with a sensitive detection technique. At present, numerous separation techniques, including high-performance liquid chromatography (HPLC) [10], gas chromatography (GC) $[11,12]$ and capillary electrophoresis (CE) $[13,14]$, have been used for the analysis of psychotropic drugs. Among those methods, HPLC has been regarded as the most efficient and influential specific technique to some advantages including convenience, high separation ability and simple operation.

Because of the complex matrix of biological fluids and low concentrations of CLO in these samples, the extraction and preconcentration of CLO is the main challenge in the sample preparation step. In this regard, microextraction methods may be useful, with major trends toward simplification, miniaturization, and minimization of the organic solvents used. Typically, microextraction techniques are grouped into SPME and LPME. SPME is a powerful sample preparation method combining the microextraction and preconcentration of a solute in a single step [15-18]. SPME has been applied mainly to the extraction of volatile and semivolatile compounds from various biological fluids by coupling the technique to GC [17-20]. LPME, on the other hand, is a miniaturized version of LLE, in which a few drops of solvent suffice to concentrate chemical species from various samples rather than large volumes required in traditional LLE. It is compatible with capillary GC, CE, and HPLC [21].

Among LPME methods, dispersive liquidliquid microextraction (DLLME) [22] succeeded in attracting considerable attention from researchers because of its fast, simple, and efficient extraction procedure, and direct injection of the extracted phase to various instruments. An appropriate mixture of extracting and disperser solvents is rapidly injected into a conical test tube containing aqueous solution, therefore, a cloudy solution 
is created. At this time, the analyte in the aqueous solution is extracted into fine droplets of extracting solvent. During centrifugation, the extracted phase will settle at the bottom of the tube, which will then be isolated. Up to now, DLLME has been successfully applied to the extraction of different families of organic and inorganic compounds from different matrices [23-25], with several reviews being written on this issue [26-28].

Despite the many advantages of DLLME, the high density of the extraction solvents is considered to be its main drawback. The solvents, being mostly halogenated, are generally dangerous for the laboratory personnel and sometimes are not compatible with RP-HPLC. Therefore, extraction solvents lighter than water were used in DLLME, adopting special designs such as narrow-neck glass tubes [29] etc., but complete removal of the collected phase was difficult or impossible in some cases. The other drawback of lighter extraction solvents is their exposure to air after performing DLLME, limiting the use of low-boiling-point solvents.

DLLME-SFO, the new and novel method of liquid-liquid microextraction integrated with the solidification of a floating organic drop (DLLME-SFO) [30] has overcome the above mentioned drawbacks. It is based on an organic extractant that has lower density than water, but solidifies at near ambient temperature. After centrifugation, the test tube is made cool by inserting it into an ice bath. The enriched analyte in the floated solid phase is collected simply by a spatula and melted at room temperature; then, it is eventually determined by chromatography or

Figure 1. Chemical structure of clozapine spectrometry methods [31].

Recently, new DLLME techniques have been introduced to eliminate dispersive solvents: air assisted (AA-DLLME), vortex assisted (VA-DLLME) and ultrasound assisted (US-DLLME). Recent literature reports the comparison between AA-DLLME, VA-DLLME and UA-DLLME [32]. Although AA-DLLME must be manually performed; it demonstrates lower centrifugation time, less analysis time without relevant differences in enrichment factor and recovery.

The aim of present study is to develop AADLLME-SFO as a fast, simple, precise, and reliable method for determination of CLO in human serum sample. After extraction of CLO from human serum sample under optimized conditions, the extracted phase was directly injected into HPLC-UV for analysis.

\section{Experimental}

\section{Chemicals and reagents}

Pure standard powder of CLO was kindly provided by TEMAD (Tehran, Iran). Sodium chloride, sodium dihydrogen phosphate, boric acid, sodium hydroxide, ethanol, acetonitrile, methanol (HPLC grade), 1-undecanol, and 1dodecanol were purchased from Merck (Darmstadt, Germany). The ultrapure water (six times distilled) was purchased from Shahid Ghazi Pharmaceuticals (Tabriz, Iran). Working solution of CLO was prepared daily in ultrapure water.

Working solution of CLO was prepared daily in ultrapure water. Blank human serum samples (drug free) were provided by healthy volunteers in our lab (as match matrices for<smiles>CN1CCN(C2=Nc3cc(Cl)ccc3Nc3ccccc32)CC1</smiles> 
drawing the calibration curves). Human serum samples were taken from a young man who was under CLO treatment and stored in polypropylene tubes at $-2{ }^{\circ} \mathrm{C}$ before use. All solutions and frozen biological samples were thawed at ambient temperature before use.

\section{Instrumentation and chromatographic conditions}

The HPLC system (Shimadzu, Kyoto, Japan) included two pumps (LC-10AD), a column oven (CTO-10A), UV-Vis spectrophotometer detector (SPD-10AD) operated at wave-length of $254 \mathrm{~nm}$, degasser (DGU-3A), and data processor (C-R4A) all from Shimadzu. Chromatographic separation was obtained using a Shim-pack CLC-ODS analytical column (150 $\mathrm{mm} \times 4.6 \mathrm{~mm}$ id), which was packed with $5 \mu \mathrm{m}$ particles and a Shim-pack G-ODS guard column $(1 \mathrm{~cm} \times 4.0 \mathrm{~mm}$ id, $5 \mathrm{~m}$ particle size $)$ with a mobile phase consisting of acetonitrile/phosphate buffer (40:60, v/v), adjusted to $\mathrm{pH} 3.5$ with phosphate buffer $(0.1$ $\mathrm{M})$. The temperature of column oven was set at $31{ }^{\circ} \mathrm{C}$, as was optimized in the previous work [33]. Short retention times result in sharper and more symmetric peaks, and provide better resolution, which are some of the benefits of the use of elevated temperatures. The mobile phase was filtered, degassed, and then pumped to the column at a flow rate of $1.5 \mathrm{~mL} / \mathrm{min}$ with a backpressure of $150 \mathrm{~kg} / \mathrm{cm}^{2}$. Extracted sample solutions including CLO were injected to the column under the above conditions. The column was washed with a stream of methanol at a flow rate of $0.5 \mathrm{~mL} / \mathrm{min}$ after each experiment. $\mathrm{PH}$ measurements were performed using a Jenway 3345 Model $\mathrm{pH}$ meter with a combined glass electrode.

\section{Sample preparation}

\section{Removal of proteins from serum samples}

According to the method of Han et al., [34], $1 \mathrm{~mL}$ of human serum sample was placed in a glass tube and a mixture $(0.9 \mathrm{~mL})$ of $15 \% \mathrm{w} / \mathrm{v}$ zinc sulfate solution/acetonitrile was added. The glass tube was vortexed for $20 \mathrm{~min}$, then maintained at $4{ }^{\circ} \mathrm{C}$ for $15 \mathrm{~min}$ followed by a centrifugation at $4000 \mathrm{rpm}$ for $5 \mathrm{~min}$. The supernatant was then collected in a sample tube and diluted to $5 \mathrm{~mL}$ using pure water. The final solution was then subjected to AADLLME-SFO procedure.

\section{AA-DLLME-SFO procedure}

To the prepared human serum sample in a $15 \mathrm{~mL}$ centrifuge tube which contained CLO $(5.0 \mathrm{~mL})$ in acetate buffer $(0.1 \mathrm{M}, \mathrm{pH} 4)$, extraction solvent (1-undecanol, $40 \mu \mathrm{L})$ was injected immediately using a syringe (gastight, Hamilton, Reno, NV, USA). To form a cloudy solution, the mixture was rapidly pulled into a $10 \mathrm{~mL}$ glass syringe from the tube with a little air and then pushed out into it (repeated eight times) via syringe needle. A cloudy mixture was created with dispersion of fine organic droplets into the bulk sample solution. At this step, CLO molecules were extracted into 1-undecanol in a few seconds. After centrifugation at $5000 \mathrm{rpm}$ for $5 \mathrm{~min}$, because of the low density of the droplets, the tiny organic droplets were floating at the top of the glass tube. To solidify the floating organic droplets which had an m.p. of $19{ }^{\circ} \mathrm{C}$, the glass tube was then cooled in an ice bath for $5 \mathrm{~min}$. The solidified droplets were easily transferred to a conical vial using a small spatula in which it melted rapidly at room temperature. Subsequently, the extractant was injected into HPLC-UV system for analysis.

\section{Optimization of AA-DLLME-SFO procedure}

Several experimental parameters affecting the extraction of CLO from human serum, like the type and volume of extraction solvents, the number of extraction cycle, $\mathrm{pH}$, and ionic strength, were assessed to optimize the AADLLME-SFO process. The optimization 
procedure was done by recording the chromatographic peak area for the samples spiked with CLO under different experimental conditions. Based on preoptimization results of the extraction parameters, the effect of each parameter was investigated using factor by factor approach, i.e., one parameter differed while the other parameters were fixed at the preselected values. Some of the important features of AA-DLLME-SFO at optimized conditions were evaluated based on the following definitions.

The EF was defined as the ratio between the solute concentration in the floated phase $\left(\mathrm{C}_{\text {flo }}\right)$ and its initial concentration $\left(\mathrm{C}_{0}\right)$ within the sample,

$$
\mathrm{EF}=\mathrm{C}_{\text {flo }} / \mathrm{C}_{\mathrm{o}}
$$

The extraction recovery (ER \%) was defined as the ratio between the number of moles of the solute in the floating phase $\left(\mathrm{n}_{\mathrm{flo}}\right)$ and initial amount $\left(\mathrm{n}_{\text {o) }}\right.$ within the sample,

$\mathrm{ER} \%=\frac{n_{f l o}}{n_{o}} \times 100=\frac{C_{f l o} V_{f l o}}{C_{o} V_{\text {sample }}} \times 100$

Where $V_{\text {flo }}$ and $V_{\text {sample }}$ are the volumes of the floating phase and sample, respectively. The relative recovery (RR\%) was obtained from the following equation:

$\mathrm{RR} \%=\frac{C_{\text {found }}-C_{\text {real }}}{C_{\text {added }}} \times 100$

Where $\mathrm{C}_{\text {found }}, \mathrm{C}_{\text {real, }}$, and $\mathrm{C}_{\text {added }}$ are the total concentration of the solute after the addition of a known amount of standard to real sample, the original concentration of solute in real sample, and concentration of the standard solution, which was spiked to the real sample, respectively.

\section{Results and Discussion}

Selection of the extraction solvent and its volume
Choosing the most appropriate extraction solvent is of primary importance for obtaining good extractability and selectivity of the target analytes in LLEs. In AA-DLLME-SFO, some special factors should be considered: the extraction solvent should have a melting point below room temperature, with lower density compared to water (for solidification and floating). Therefore, 1-undecanol and 1dodecanol and hexadecane were examined, which are the three most perfect organic solvents that have the above-mentioned qualities and have been used in SFO procedure. Among these, the highest extraction recovery (ER\%) for CLO was obtained by 1-undecanol (Figure 2). Therefore, 1-undecanol (m.p. $19{ }^{\circ} \mathrm{C}$ ) was selected as the proper extraction solvent.

Various volumes of 1-undecanol were tested during the optimization $(40,50,60,70$, $80,100,120 \mu \mathrm{L})$. Results indicated the decrease of the peak area with the increase of the 1-undecanol volume. It was discovered that with increase in the volume of 1undecanol from 40 to $100 \mu \mathrm{L}$, the volume of the floated phase increased from about 35 to $95 \mu \mathrm{L}$. The highest ER\% was obtained with 40 $\mu \mathrm{L}$ of the extraction solvent and decreased with volumes $>40 \mu \mathrm{L}$ (Figure 3). Poor reproducibility and difficulty in handling small volumes of solidified extractant limited the use of volumes $<40 \mu \mathrm{L}$ of 1-undecanol. Therefore, $40 \mu \mathrm{L}$ was chosen as the optimum volume of 1-undecanol as the extraction solvent for CLO in AA-DLLME-SFO procedure.

\section{Effect of the number of extraction cycle}

For the proposed method, the number of extraction was defined as the number of repeatedly sucking extraction solvent and sample solution mixture into a $10 \mathrm{~mL}$ glass syringe and then its injecting into the test tube. 
Figure 2. Effect of type of extraction solvent on the extraction recovery of CLO obtained from AA-DLLME-SFO

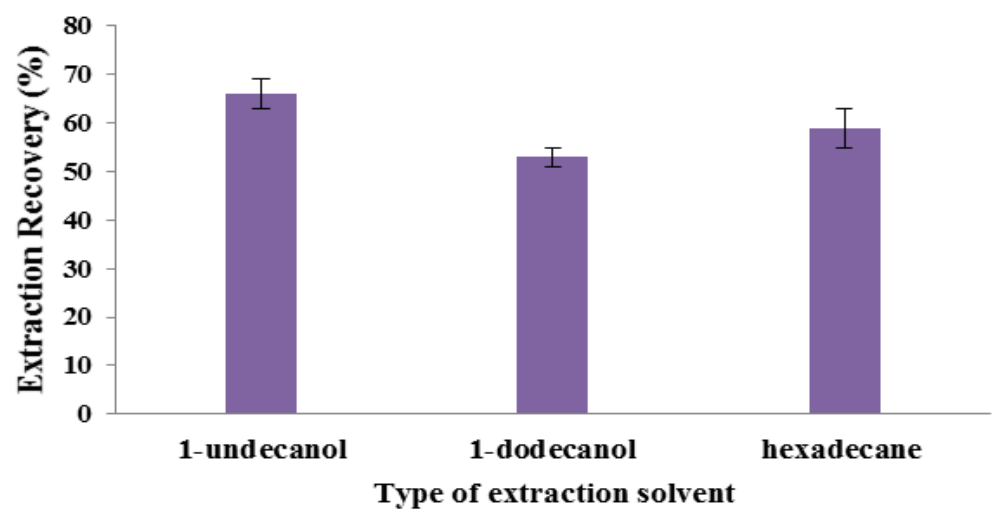

Figure 3. Effect of the volume of 1-undecanol as extraction solvent on the extraction recovery of CLO obtained from AA-DLLMESFO. Extraction conditions: sample volume $(5.0 \mathrm{~mL}) ; \mathrm{pH}$ 4 ; number of extraction cycle: 8; concentration of ATR $\quad(1000 \mu \mathrm{g} / \mathrm{L})$. All experiments were performed in triplicate $(\mathrm{n}=3)$

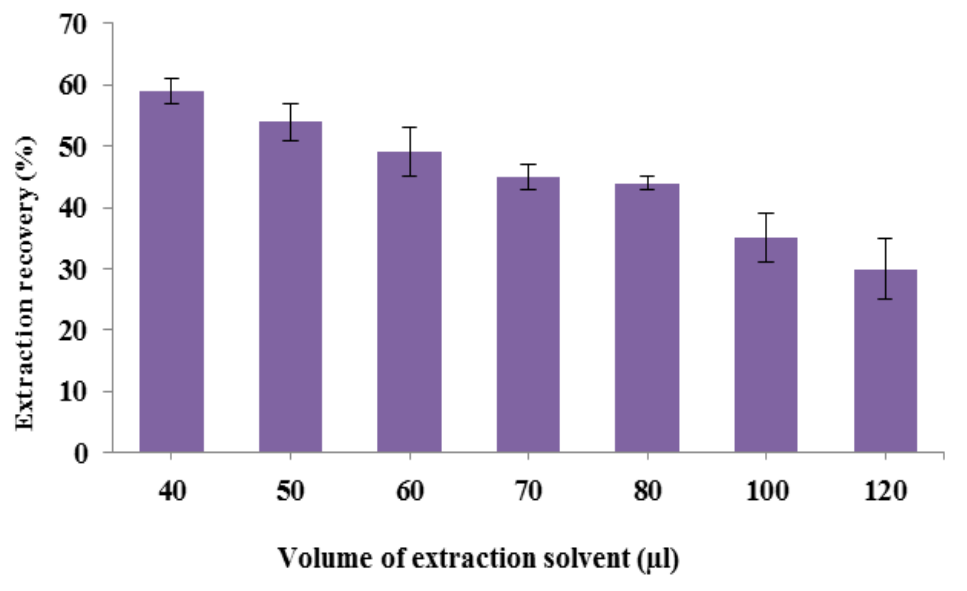

It is predictable that recoveries increase by increasing the number of extraction cycles. Ten extractions cycles was investigated. The peak areas of the analytes increased up till eight extraction cycles then a plateau was observed. Moreover by increasing the number of extraction the repeatability improved, yet in case of extraction cycles higher than eight the repeatability decreased. Hence, eight extraction cycles were selected as optimum for the AA-DLLME-SFO, it was noted that this step was performed in less than 1 min.

\section{Effect of $p H$}

The effect of $\mathrm{pH}$ on the extraction efficiency of AA-DLLME-SFO was evaluated within the range of $2-11$ for the preconcentration of CLO. Three buffers were used for covering the whole $\mathrm{pH}$ range, namely, acetate buffer ( $\mathrm{pH} 2-5)$, phosphate buffer ( $\mathrm{pH} \mathrm{6-8)}$ ) and ammonia buffer $(\mathrm{pH}$ 8-10). The highest ER for CLO had seen at $\mathrm{pH} 4$ and 8 which is near two $\mathrm{pK}_{\mathrm{a}}$ of CLO (3.7, 7.6). Generally aqueous solution should be sufficiently acidic or basic to keep the analyte in its neutral form. The positive or negative ion is less extractable to 1-undecanol than neutral CLO. Based on figure 4 the $\mathrm{pH}$ value of 4 was chosen as the optimum $\mathrm{pH}$ for extraction of CLO by AADLLME-SFO.

\section{Salt effect and extraction time}

To study the salt effect, AA-DLLME-SFO experiments were conducted at different $\mathrm{NaCl}$ concentrations, ranging from 0 to $5 \%$ $\mathrm{w} / \mathrm{v}$. The chromatographic peak area for CLO $(1000 \mu \mathrm{g} / \mathrm{L})$ was almost without 
change in the presence of $\mathrm{NaCl}$ concentrations up to $1 \% \mathrm{w} / \mathrm{v}$. Higher concentrations of $\mathrm{NaCl}$ caused the decrease in the extraction efficiency of CLO (not shown), which may be due to increased viscosity of the sample solution, thus restraining CLO transport to the extraction solvent. Consequently, the addition of salt was not advantageous in the present work. In DLLME techniques, the equilibrium state is quickly achieved, because of the fast dispersing of the extraction solvent in all of the samples, thus, the equilibration time is not a critical factor. Therefore, the interval between the time immediately after the injection of extraction solvent and the time before the centrifugation step is optimized as the extraction time. The effect of the extraction time was investigated in the range of $0.5-20 \mathrm{~min}$, and the results showed that there was no significant difference in peak areas of CLO at different extraction times. Therefore, a centrifugation step was conducted a few seconds after injection process.
The performance of AA-DLLME-SFOHPLC-UV method was evaluated (Table 1). Precision, EF, ER, linear dynamic range, correlation coefficient $\left(\mathrm{R}^{2}\right)$, and LOD were calculated. A series of 12 concentration levels of CLO were prepared (in serum samples) to establish and obtain the calibration curve. Three replicate extraction experiments at each concentration level were conducted. For the calibration curves in serum samples, high $\mathrm{R}^{2}$ values were obtained $(>0.99)$, which confirmed the adequate linearity. LODs of the method in serum samples were determined based on the concentration of CLO, which produced chromatographic peaks with $\mathrm{S} / \mathrm{N}$ of 3 . The wide linear ranges (4-2000 ng/mL) and very low LODs of the method are advantageous for CLO analysis in biological fluids. The repeatability was studied by performing five parallel replicate extractions (at concentration of $100 \mu \mathrm{g} / \mathrm{L}$ ) under the optimized conditions. The resultant repeatability expressed as RSD $\%$ was $<6.6 \%$.

\section{Analytical characteristics of the method}

Figure 4. Effect of $\mathrm{pH}$ on the extraction recovery of CLO obtained from AA-DLLME-SFO. Extraction conditions: sample volume $(5.0 \mathrm{~mL})$; number of extraction cycle: 8 , extraction solvent (1-undecanol, $40 \mu \mathrm{L}$ ); concentration of ATR $(1000$ $\mu \mathrm{g} / \mathrm{L})$. All experiments were performed in triplicate $(n=3)$

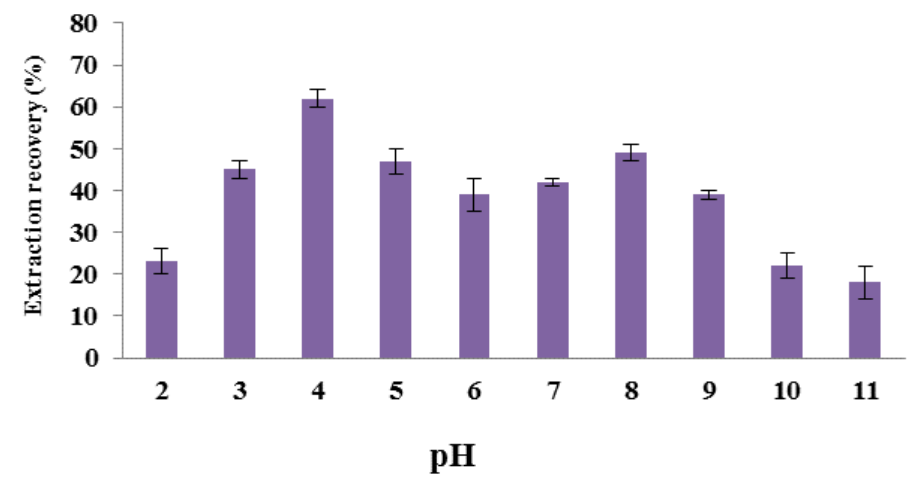

Table 1. Analytical characteristics of AA-DLLME-SFO-HPLC for determination of CLO in real samples

\begin{tabular}{lcccccc} 
sample & LOD $(\mathrm{ng} / \mathrm{mL})$ & $\mathrm{LRa}(\mathrm{ng} / \mathrm{mL})$ & $\mathrm{EFb}$ & $\mathrm{R}^{2 \mathrm{c}}$ & $\mathrm{ER}(\%)$ & $\mathrm{RSD}(\%, \mathrm{n}=5)$ \\
\multicolumn{1}{c}{1.34} & $4-2000$ & 128 & 0.995 & 97 & 6.3 \\
\hline serum Linear range & & & & & \\
a L Extraction factor \\
c Squad-correlation coefficient
\end{tabular}


A comparison was made between AADLLME-SFO-HPLC-UV with some of the similar published methods for CLO extraction and determination (Table 2). The proposed method in serum matrices has wide linearity ranges and the lowest LOD among the reported methods. Therefore, it can be used for the isolation and determination of trace amounts of CLO in complex biological fluids.

\section{Real sample analysis}

The performance and applicability of AA-DLLME-SFO for the extraction and subsequent determination of CLO by HPLCUV were examined by spiking CLO standard solutions into serum samples from a person under treatment of CLO (concentrations of spiked CLO in samples were $200 \mu \mathrm{g} / \mathrm{L}$ ). The serum samples were subjected to the proposed procedure of AA-DLLME-SFO (three replicate measurements for each blood serum sample). To validate the method two different concentrations of CLO were added to the actual serum samples and the recommended procedure was applied.

The results of RR and the concentrations obtained are included in Table 3. Figure 5 shows typical chromatograms in which blank serum samples showed no distinguishable chromatographic peak at about 5 min (Figure 5A). The presence of CLO in the samples from a person consumed CLO (Figure 5B) showed the characteristic peak of the drug at about5.1 min. The increased peak height (and peak area) correlates very well with the increasing amount of CLO in the sample (Figure 5C). These results confirmed that the biological matrices in our present context had no significant effect on AADLLME-SFO-HPLC-UV for the determination of CLO.

Table 2. Comparison of AA-DLLME-SFO-HPLC-UV with other extraction methods for determination of CLO

\begin{tabular}{|c|c|c|c|c|}
\hline $\begin{array}{l}\text { Extraction and determination } \\
\text { technique }\end{array}$ & matrix & $\mathrm{LR}^{\mathrm{a}}(\mathrm{ng} / \mathrm{mL})$ & $\begin{array}{c}\mathrm{LOD}^{\mathrm{b}} \\
(\mathrm{ng} / \mathrm{mL})\end{array}$ & Ref. \\
\hline RP-HPLC & serum & $5-1000$ & 5 & Zaren et al. \\
\hline SPE-HPLC-DAD ${ }^{c}$ & serum & $10-1000$ & 3.15 & Petruczynik et al. \\
\hline SPE-HPLC-DAD & saliva & $10-1000$ & 5.65 & Petruczynik et al. \\
\hline SPE-HPLC-UV & plasma & $20-2500$ & 7 & Wheeler et al. \\
\hline $\mathrm{GC}$ & serum & $3-300$ & $1-2$ & Richter \\
\hline HPLC-ED & plasma & $50-600$ & 20 & Humpel et al. \\
\hline TDLLME-HPLC & plasma & $5-3000$ & 1.5 & Asghari et al. \\
\hline UA-LDS-DLLME & blood & $5-500$ & $\mathrm{LLOQ}=5$ & Chen et al. \\
\hline DLLME-HPLC & urine & $50-5000$ & 25 & Chen et al. \\
\hline AA-DLLME-SFO-HPLC-UV & serum & $4-2000$ & 1.3 & This work \\
\hline
\end{tabular}


Figure 5. (A) Blank serum sample; (B) real serum sample from a person under clozapine treatment; (C) A + 200 $\mu \mathrm{g} / \mathrm{L}$ of CLO. Extraction conditions: sample volume $(5.0 \mathrm{~mL})$; extraction solvent (1undecanol, $\quad 40 \mu \mathrm{L}$ ); number of extraction cycle: $8 ; \mathrm{pH}$ 4. All experiments were performed in triplicate $(\mathrm{n}=3)$

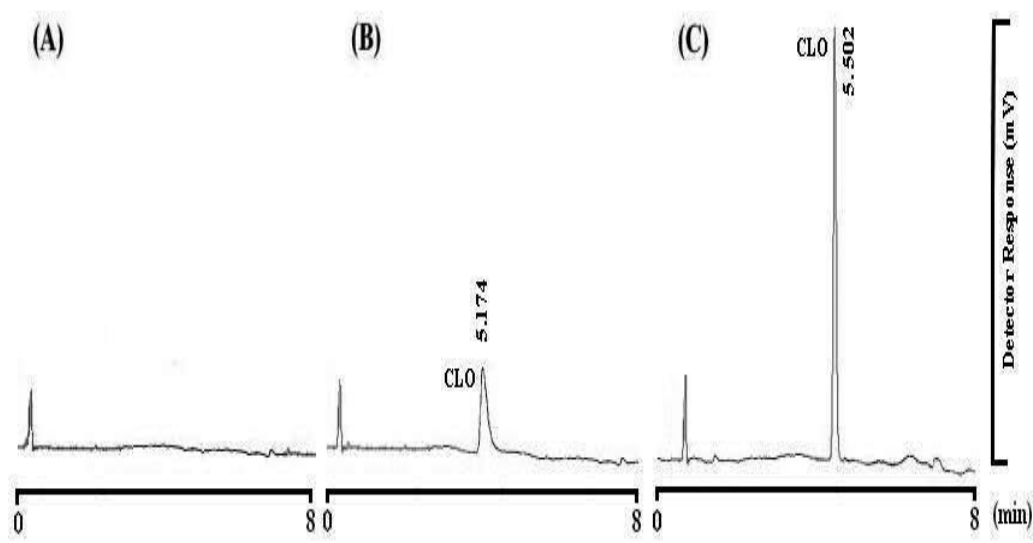

Table 3. Analysis of human serum samples for CLO by the proposed methoda

\begin{tabular}{cccc} 
Compound & CLO added $(\mu \mathrm{g} / \mathrm{l})$ & Found $\left( \pm \mathrm{SD}^{\mathrm{b}}, \mathrm{n}=3\right)(\mu \mathrm{g} / \mathrm{l})$ & $\mathrm{RR}(\%)$ \\
Clozapine & 0 & $95.4(8.7)$ & - \\
& 100 & $209.5(12.5)$ & 114.1 \\
& 300 & $408.7(14.1)$ & 105.8 \\
\hline
\end{tabular}

a These data are based on the diluted volumes of human serum samples and dilutions effect was considered for calculation of the concentrations

b Standard deviation

\section{Conclusion}

In the present study, AA-DLLME-SFO, as a sample and novel pretreatment technique, was coupled to HPLC-UV method for the quantification of clozapine in serum samples. A low-density and low-toxicity organic solvent (1-undecanol) was utilized as extraction solvent and the extractant droplets were collected easily as supernatant by solidifying at $19{ }^{\circ} \mathrm{C}$. Furthermore, the SFO solvent facilitated the phase separation. The method was simple, rapid, and reproducible. The calibration plot was linear over a wide concentration range of clozapine with a very low LOD of 1.3 $\mathrm{ng} / \mathrm{mL}$. The established method was applied to the determination of low concentrations of clozapine in real serum samples. The experimental results revealed that AADLLME-SFO-HPLC-UV provides good accuracy and precision for the determination of low concentration levels of clozapine within a short analysis time.

\section{Acknowledgments}

The research council of Islamic Azad University, Najafabad branch is gratefully acknowledged for their financial support. The authors also acknowledged the TEMAD (Tehran, Iran) for providing pure CLO.

\section{Disclosure statement}

No potential conflict of interest was reported by the authors.

\section{References}

[1] S.W. Benson, The Foundations of Chemical Kinetics; McGraw-Hill: New York, 1960, p 342.

[2] S.W. Lewis, T.R.E. Barnes, L. Davies, R.M. Murray, G. Dunn, K.P. Hayhurst, A. 
Markwick, H. Lloyd, P.B. Jones, Schizophr. Bull., 2006, 32, 715-723.

[3] L. Voruganti, L. Cortese, L. Oyewumi, Z. Cernovsky, S. Zirul, A. Awad, Schizophr. Res., 2000, 43, 135-145.

[4] J. Hennen, R.J. Baldessarini, Schizophr. Res., 2005, 73, 139-145.

[5] A.M. Walker, L.L. Lanza, F. Arellano, K.J. Rothman, Epidemiology, 1997, 8, 671677.

[6] A. Wheeler, V. Humberstone, G. Robinson, J. Psychopharm., 2009, 23, 957-965.

[7] S.M. Stahl, Psychopharmacology of antipsychotics, Taylor \& Francis, 1999.

[8] D.E. Casey, Psychopharmacology, 1989, 99, S47-S53.

[9] J.M. Kane, T.B. Cooper, E.J. Sachar, F.S. Halpern, $\mathrm{S}$.

Bailine, Psychopharmacology, 1981, 73, 184187.

[10] M. Hasegawa, P.A. Cola, H.Y. Meltzer, Neuropsychopharmacology, 1994, 11, 45-48.

[11] C. Xiong, J. Ruan, Y. Cai, Y. Tang, J. Pharm. Biomed. Anal., 2009, 49, 572578.

[12] H.C. Huang, C.H. Liu, T.H. Lan, T.M. Hu, H.J. Chiu, Y.C. Wu, Y.L. Tseng, J. Chromatog. B, 2007, 856, 57-61.

[13] C. Sasaki, T. Shinozuka, C. Murakami, W. Irie, K. Maeda, T. Watanabe, N. Nakamaru, M. Furukawa, S. Nakamura, K. Kurihara, Forensic Sci. Int., 2013, 227, 90-94.

[14] S. Dziomba, P. Kowalski, T. Bączek, J. Pharm. Biomed. Anal., 2012, 62, 149154.

[15] Li, J., F. Zhao, H. Ju, Anal. Chim. Acta, 2006, 575, 57-61.

[16] C.L. Arthur, J. Pawliszyn, Anal. Chem., 1990, 62, 2145-2148.

[17] A.R. Chaves, G.C. Júnior, M.E.C. Queiroz, J. Chromatog. B, 2009, 877, 587-593.

[18] M. Queiroz, C. Valadão, A. Farias, D. Carvalho, F. Lanças, J. Chromatog. B,
2003, 794, 337-342.

[19] B.J.G. Silva, F.M. Lanças, M.E.C. Queiroz, J. Chromatog. B, 2008, 862, 181-188.

[20] M.D. Oliveira, M.E.C. Queiroz, D. Carvalho, S. Silva, F. Lanças, Chromatographia, 2005, 62, 215-219.

[21] M. Tankiewicz, C. Morrison, M. Biziuk, Talanta, 2013, 107, 1-10.

[22] A. Sarafraz-Yazdi, A. Amiri, TrAc, Trends Anal. Chem., 2010, 29, 1-14.

[23] M. Rezaee, Y. Assadi, M.R.M. Hosseini, E. Aghaee, F. Ahmadi, S. Berijani, J. Chromatog. A, 2006, 1116, 1-9.

[24] X.W. Lai, D.L. Sun, C.Q. Ruan, H. Zhang, C.L. Liu, J. Sep. Sci., 2014, 37, 92-98.

[25] S. Li, P. Gao, J. Zhang, Y. Li, B. Peng, H. Gao, W. Zhou, J. Sep. Sci., 2012, 35, 3389-3395.

[26] M.T. Naseri, P. Hemmatkhah, M.R.M. Hosseini, Y. Assadi, Anal. Chim. Acta, 2008, 610, 135-141.

[27] V. Andruch, I.S. Balogh, L. Kocúrová, J. Šandrejová, Appl. Spectrosc. Rev., 2013, 48, 161-259.

[28] J.M. Kokosa, TrAc, Trends Anal. Chem., 2013, 43, 2-13.

[29] Y. Santaladchaiyakit, S. Srijaranai, R. Burakham, J. Sep. Sci., 2012, 35, 23732389.

[30] P. Hashemi, S. Beyranvand, R.S. Mansur, A.R. Ghiasvand, Anal. Chim. Acta, 2009, 655, 60-65.

[31] M.I. Leong, S.D. Huang, J. Chromatog. A, 2008, 1211, 8-12.

[32] F.R. Mansour, N.D. Danielson, Talanta, 2017, 170, 22-35.

[33] M. Rahmani, M. Kaykhaii, Z. Safari, 0. Ansari, S. Jaberi Nasab, Chromatographia, 2018, 80, 109-117.

[34] L. Mercolini, F. Bugamelli, E. Kenndler, G. Boncompagni, L. Franchini, M.A. aggi, J. Chromatog. B, 2007, 846, 273-280.

[35] J.H. Suh, Y.Y. Lee, H.J. Lee, M. Kang, Y. Hur, S.N. Lee, D.-H. Yang, S.B. Han, J. Pharm. Biomed. Anal., 2013, 75, 214219. 
[36] W. Zeren, L. Minglian, X. Peipei, Z. Yanlin, Biomed. Chromatog., 1986, 1 , 53-57.

[37] A. Petruczynik, K. Wróblewski, M. Szultka-Młyńska, B. Buszewski, H. Karakuła-

Juchnowicz, J. Gajewski, J. MorylowskaTopolska, M. Waksmundzka-Hajnos, J. Pharma. Biomed. Anal., 2016, 127, 6880.

[38] K. Richter, J. Chromatog. B Biomed. Sci. Appl., 1988, 434, 465-468.
[39] C. Humpel, C. Haring, A. Saria, J. Chromatog. B Biomed. Sci. Appl., 1989, 491, 235-239.

[40] A. Asghari, E. Fahimi, M. Bazregar, M. Rajabi, L. Boutorabi, J. Chromatogr. B, 2017, 1052, 51-59.

[41] X. Chen, S. Zheng, J. Le, Z. Qian, R. Zhang, Z. Hong, Y. Chai, J. Pharma. Biomed. Anal., 2017, 142, 19-27.

[42] J. Chen, C. Xiong, J. Ruan, Z. Su, J. Huazhong Univ. Sci. Technolog. Med. Sci., 2011, 31, 277-284.

How to cite this manuscript: Amir Hosein Mohammad Shafiee, Mohammd Reza Mohammad Shafiee, Determination of Clozapine by Air Assisted Dispersive Liquid-Liquid Microextraction Based On Solidification of Organic Droplet Followed by HPLC in Human Serum, Adv. J. Chem. A, 2020, 3(2), 111-121. 